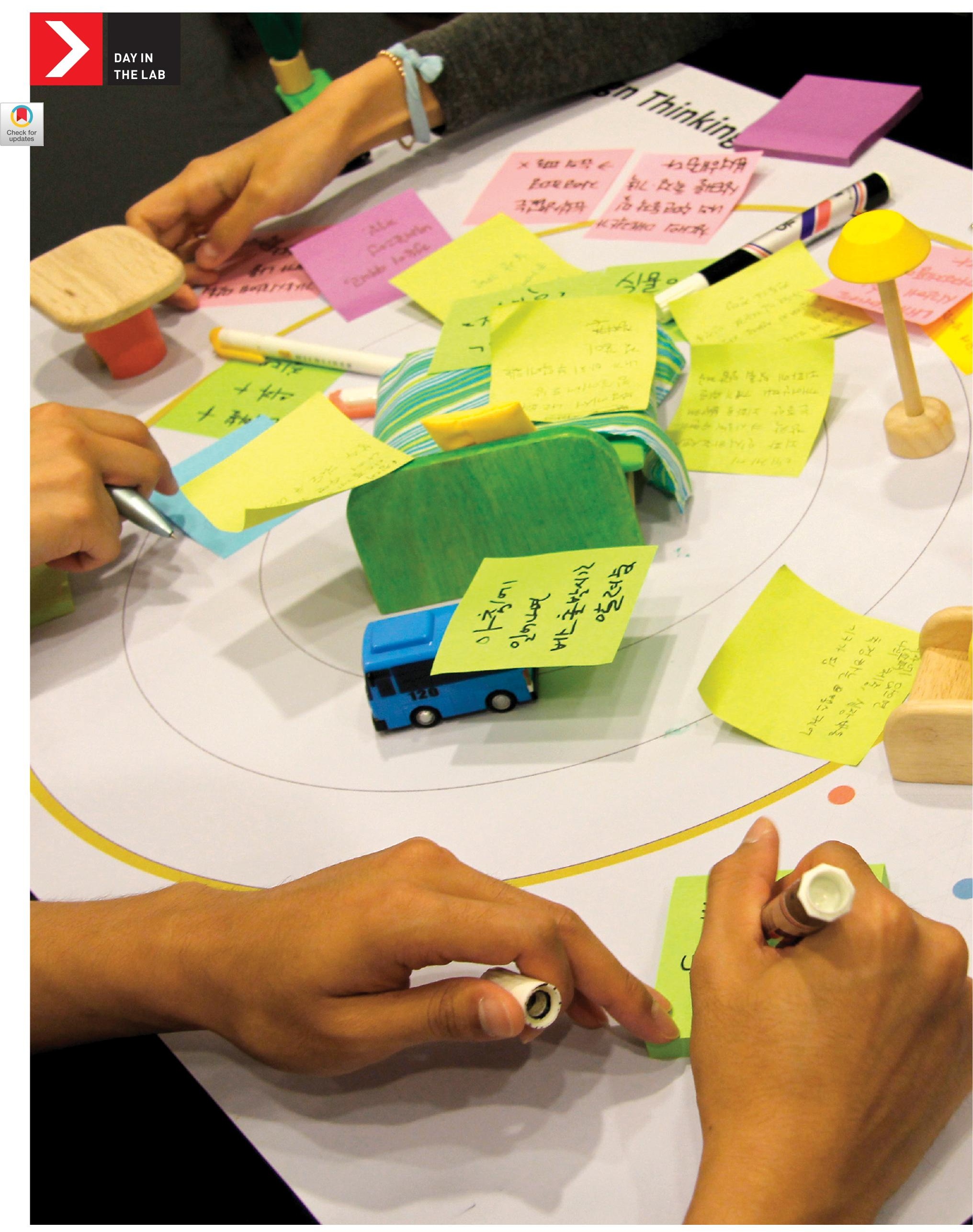




\section{Imagine Lab, Hanyang University}

As told by Ahreum Lee, Kyoungwon Seo, Jieun Kim, Gyu Hyun Kwon, and Hokyoung Ryu

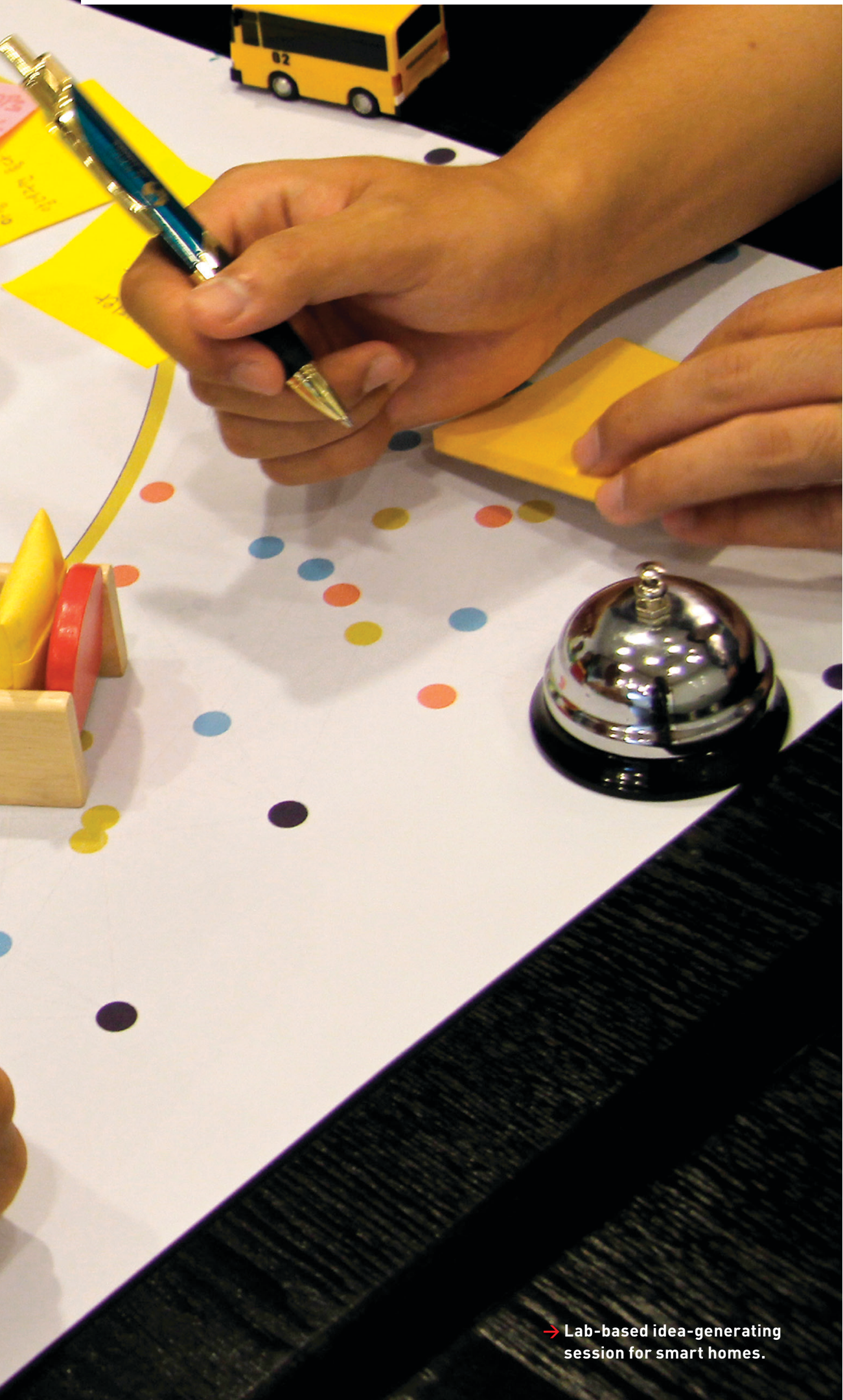

H ow do you describe your lab to visitors? Inspired by the George Bernard Shaw quote, "Imagination is the beginning of creation," Imagine Lab is a research institute that strives to explore every imagined possibility.

It was established in 2012 at

Hanyang University in Seoul, Korea, initially created by two departments, the Department of Industrial

Engineering and the Graduate School of Technology \& Innovation Management. In 2015 it expanded to include the Department of Arts \& Technology. The lab space itself is currently distributed among these three locations.

Our major research interests are arts and technology, cognitive informatics, design-driven innovation, and medical HCI.

What is a unique feature of your lab? Imagine Lab is equipped with several research platforms, including a foursided 3D Cave Automatic Virtual Environment (CAVE) system, highend 3D printers, physiological and behavioral measuring instruments, and a usability room to investigate research questions in HCI.

Imagine Lab conducts several transdisciplinary research projects. For instance, with the 3D CAVE and a tracking camera system, we built an immersive virtual reality platform. Using this platform, we assessed an individual's behavioral data to detect mild cognitive impairment, autism spectrum disorder, and so on. For this work we partnered with two other groups, the Department of Dance and the Department of Psychiatry. This interdisciplinary research, particularly with medical staff, has been possible due to the proximity of our lab and Hanyang University Medical School-a 10-minute walk. This benefit makes it easy for us to meet with various clinicians and patients. We currently work with the Departments of Gastrointestinal Internal Medicine, Rehabilitation Medicine, Neurology, Pediatrics, and Rheumatology. From this collaboration we developed RehabMaster, a virtual reality rehabilitation system for post-stroke patients, now commercialized and being used by family doctors in Korea.

Our partnership with the 

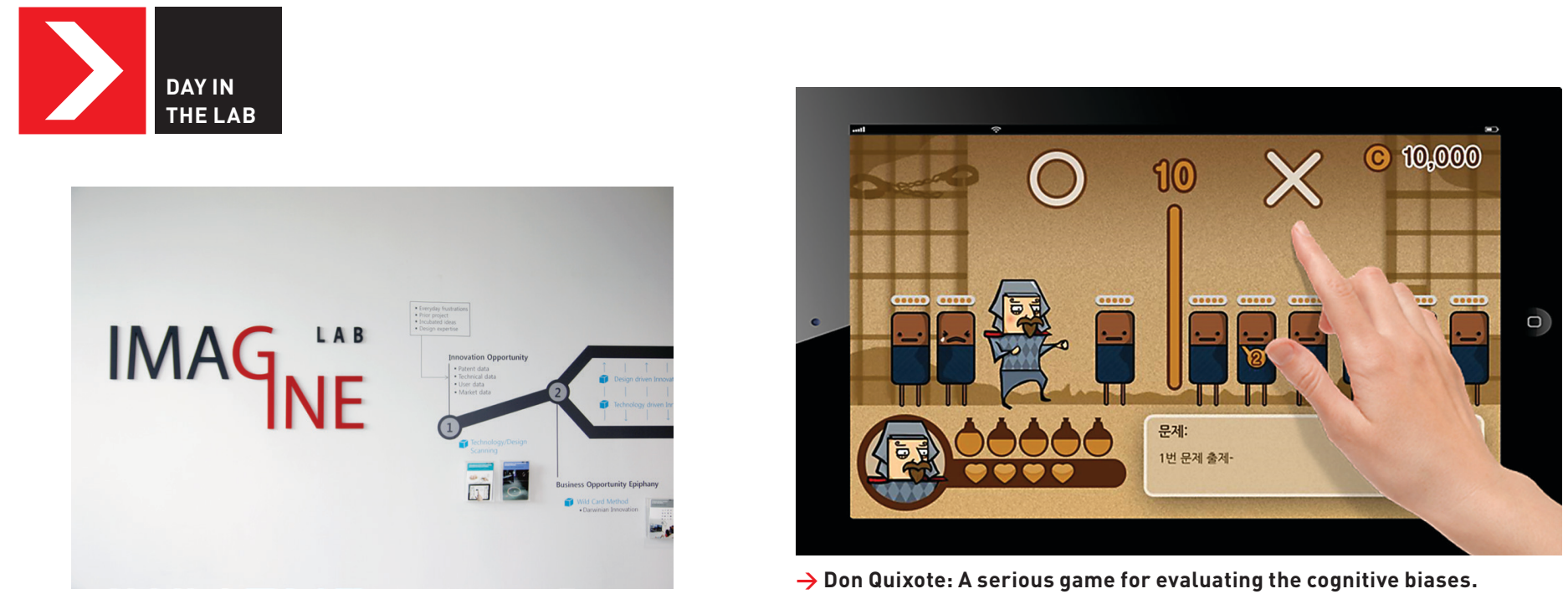

$\rightarrow$ Don Quixote: A serious game for evaluating the cognitive biases.

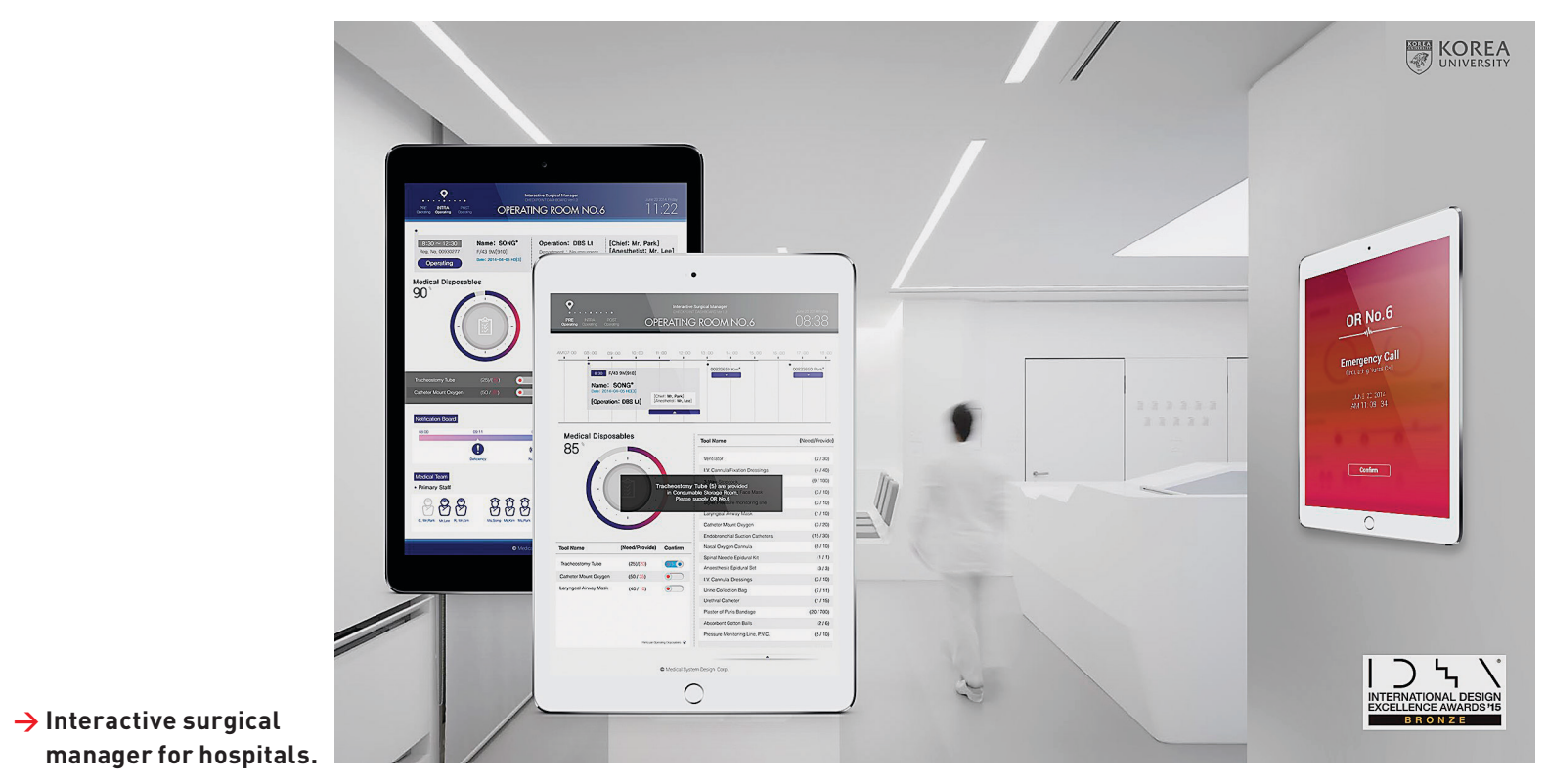

Department of Dance might sound odd, but it makes sense for us. Hanyang University is one of Korea's top private universities, home to 120 departments under 25 colleges, and hosting about 36,000 students. This diversity helps Imagine Lab lead innovative, imaginative design processes with artists, scientists, and engineers. $\mathrm{A}$ newly established department called the Department of Arts \& Technology (which includes many academic staff from music, dance, fashion, mechanics, and informatics) was the biggest addition to Imagine Lab in 2015.

How many people are in the lab, and what is the mix of backgrounds and roles? Our lab is supervised by three full-time professors (Jieun Kim, Gyu Hyun Kwon, and Hokyoung Ryu) and two full-time research professors (Eunju Jeong and Jaekwan Kim). Everyone has widely different backgrounds: cognitive psychology, industrial engineering, design, music, and bio-informatics. Currently, two Ph.D. students, eight master's students, and three undergraduate students are working together. There are also 17 part-time graduate students who work in diverse fields (electronics, automobiles, and finance, to name a few). More recently, newcomers from various fields such as music therapy, statistics, accounting, and business management have joined our lab. These unexpected arrivals have inspired us to expand our imagination to incorporate wider and more varied viewpoints.

What is one feature of your lab you want and do not have? Green space! Seoul is the most densely populated city in Korea. Most of the campuses in Seoul do not have enough green spaces for students to relax, and neither does Hanyang University. Luckily, however, we are a 10-minute drive from the Han River and a five-minute walk from Seoul Forest, which both provide some relief from the gray buildings in our daily lives.

\section{How would you describe how people} interact in your lab? The lab culture in Korea is quite strict and maledominant. However, the atmosphere in Imagine Lab provides flexibility and openness. What's more, we have an equal number of male and female students. To share feedback on individual research projects in a relaxed atmosphere, at least once a week we 

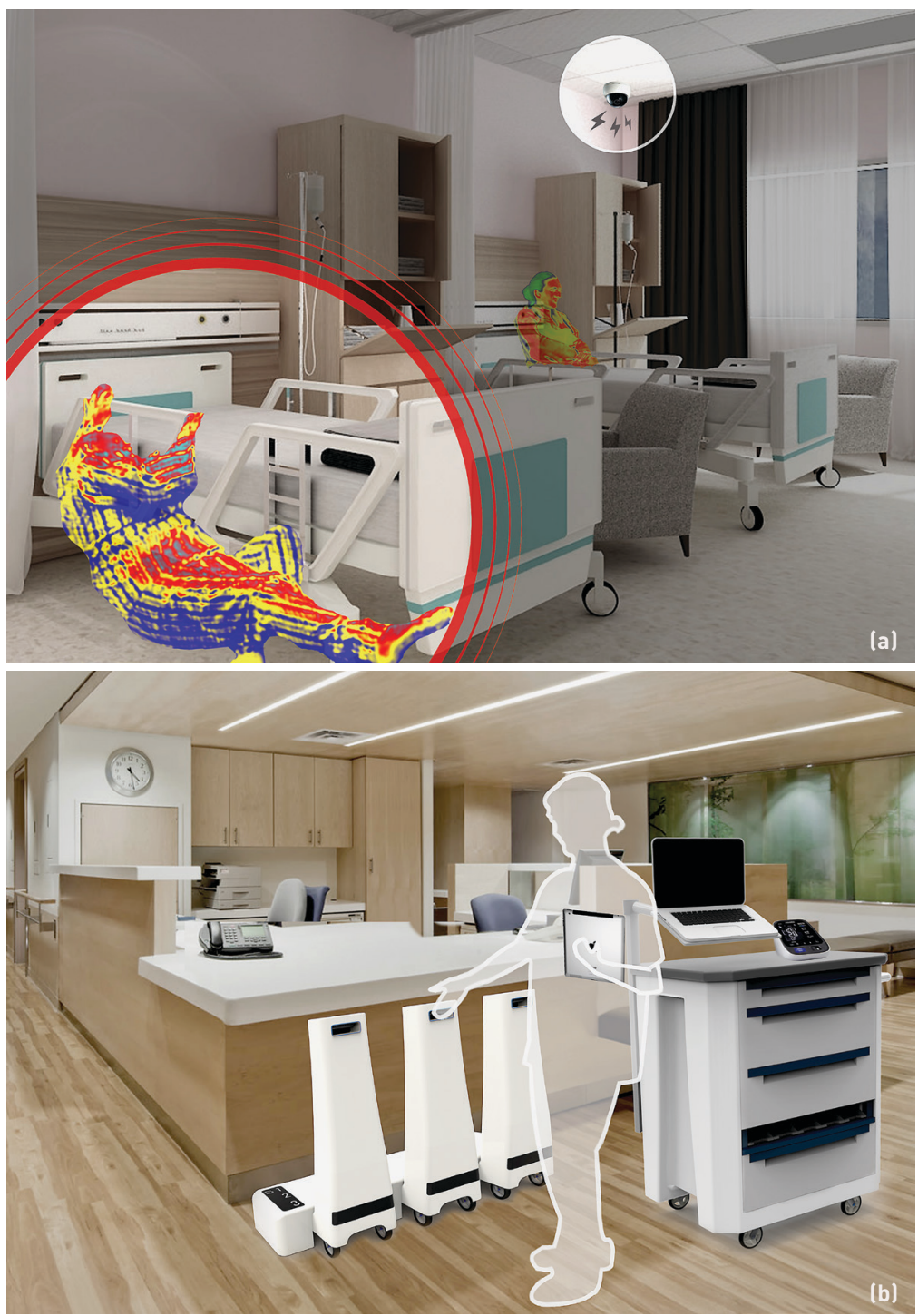

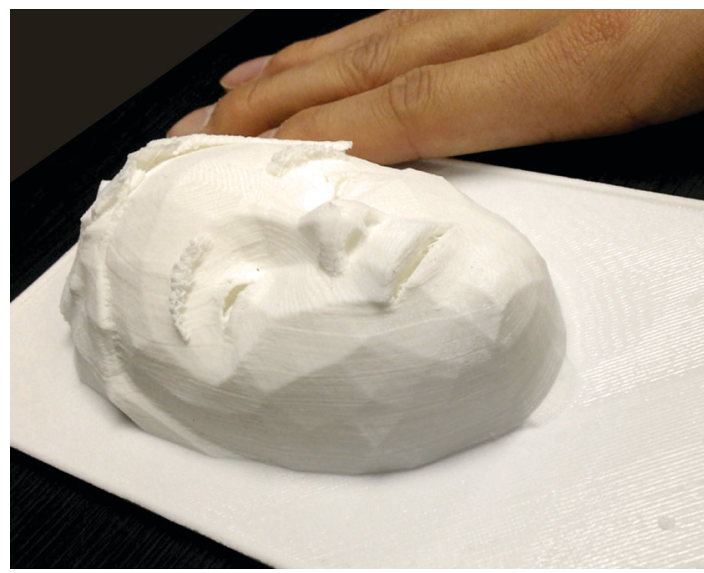

$\rightarrow$ Designing a tactile picture for the visually impaired.

\section{At Imagine Lab we do not limit the scope of our research interests.}

drink coffee and eat together. We also take walks to the Han River, which is a great way to relax and strengthen the bonds between us.

\section{What is the one thing you see as most} important about the work you do there? At Imagine Lab we do not limit the scope of our research interests. We can work on medical projects, or with dancers, or even with athletes. This extraordinary opportunity allows us to incorporate innovative and practicable viewpoints into our theoretical research that traditional HCI may have overlooked. Therefore, having an open mind as well as a sense of humor and good conversation skills are essential to working in our lab.

() http://imagine.hanyang.ac.kr

() http://artech.hanyang.ac.kr

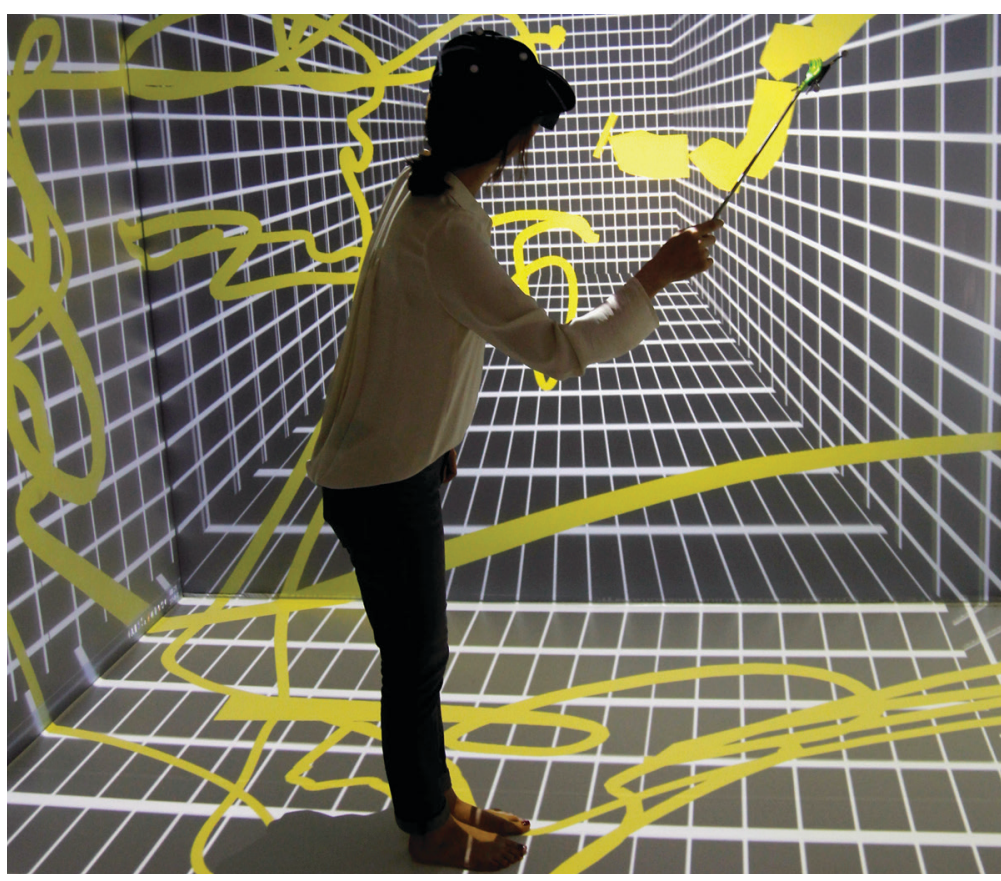

$\rightarrow$ Creative 3D drawing with artists in an immersive virtual reality environment. 